\title{
Cluster based Energy Efficient Sensing for Cognitive Radio Sensor Networks
}

\author{
Usman Mansoor \\ Department of Telecom Engineering \\ ICT Islamabad affiliated with UET Peshawar
}

\author{
Muhammad Khalil Shahid \\ Associate Professor ICT \\ PTCL Academy Islamabad
}

\begin{abstract}
In this paper, a wireless cognitive radio sensor network is considered, where each sensor node is equipped with cognitive radio. As energy consumption is the main problem when using sensors therefore a new clustering algorithm is developed according to which group of nodes form cluster having a single cluster head. Each cluster has balanced energy which prolongs overall lifetime of CRSN. Cluster heads are rotated, depending on a threshold value, in such a way as to improve the lifetime of a cluster. As new cluster head is selected immediately whenever energy of old cluster head drops to certain threshold thus improves sensing results by CRSN nodes with minimum number of faulty decisions. Simulation results demonstrate working of schemes proposed and compares the pros and cons of each scheme.
\end{abstract}

\section{Keywords}

Balanced energy, clustering algorithm, cluster head and cluster head rotation.

\section{INTRODUCTION}

Cognitive Radio Sensor Network has recently attracted a large amount of attention due to its advantage of dynamic spectrum access which provides reliability in terms of communication and enhances energy conservation potential in sensor network. Each node in a [1] [2] CSRN is typically equipped with a sensors having cognitive capability for communication purpose. Preserving the consumed energy of each node is an important goal that must be considered when developing any protocol for cognitive radio sensor networks. To satisfy these requirements, different solutions have been proposed in the past that exploit the tradeoffs among energy, accuracy, and latency. Among many solutions one of the solutions uses hierarchical architectures [3], where sensor nodes equipped with cognitive radios are clustered according to applicationspecific parameters. Sensors in cluster networks can then cooperate to sense and process a physical phenomenon. Clustering of sensors [4] helps in scalability of network and conserves energy to a large extent. Much of work in this regard has been done.

In a centralized approach every sensor sends data to the base station and thus consumes large amount of energy as the transmission distance is much larger. Using clustering approach, firstly data is transmitted to cluster head of each cluster and then that data is further transferred to the base station. Therefore much of the work load of base station distributes between cluster heads. Other than scalability [5], clustering helps in reducing routing table information.

An important feature of sensor network is energy efficiency and to extend the network's lifetime, battery source of each sensor in a network should be intelligently used so that minimum amount of energy consumption is made. Fig.1 shows general architecture of clustered network. In this figure there are two clusters i.e. cluster 1 and cluster 2 . In each cluster circles represent non cluster head nodes and those circles which are connected to the base station are the cluster head nodes. Cluster head nodes get data from their respective non cluster head nodes and then pass it to base station. Base station actually is the main centralized entity where all the data is present.

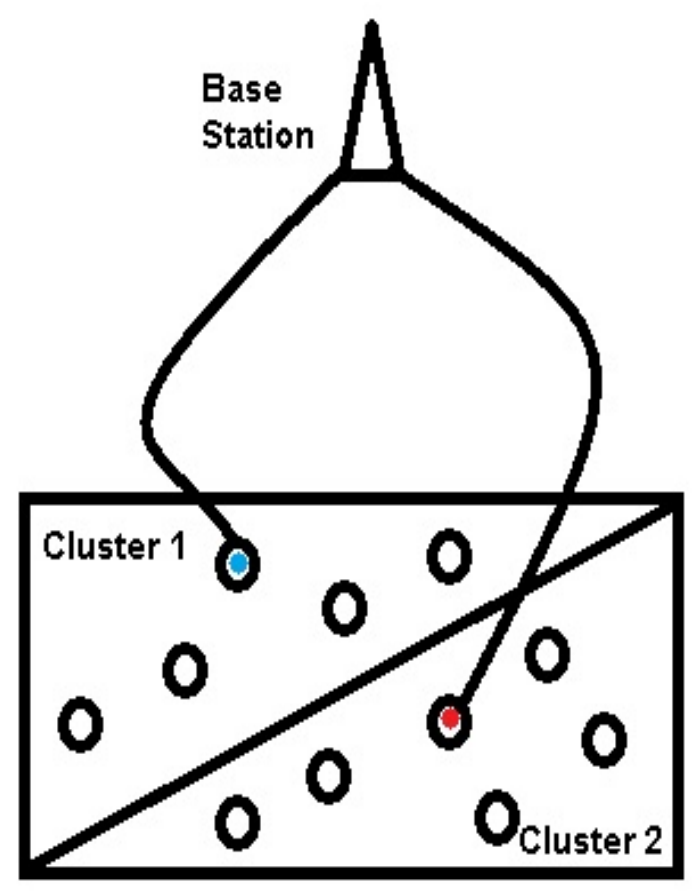

Fig 1: Hierarchical Architecture

The aim of this study is to investigate clustering algorithm which improves the energy balancing between the clusters and also the lifetime of each sensor node. Specifically initial clustering is done on the bases of distance criterion so that cluster head uniformity is maintained throughout the network. After the initial clustering, in second stage of the algorithm de attachment of some sensor nodes occur throughout the network. This second stage helps in energy balancing throughout the network. In the third stage of the algorithm cluster head selection is done on the bases of energy.

Therefore paper is organized in a way that section 2 describes a general framework of CRSN.

The optimal method of solving this problem i.e. proposed clustering algorithm is presented in complete detail in section 3 .

In the section 4 results of some preliminary experiments are presented and section 5 concludes the paper. 


\section{SYSTEM MODEL}

In this section, firstly a general framework of a proposed cognitive radio sensor network is discussed. Then radio signal model is discussed. The following assumptions are made about the sensor nodes and the network model:

1. Nodes are distributed in a spatial region. These nodes can be represented using points in three dimensional planes (x-plane, y-plane, energy).

2. Nodes are equipped with GPS capable antennae's.

3. The base station (BS) is located inside the sensing field.

4. Distances between base station and cluster head nodes (CH's) are d1, d2, d3 ... dn

5. Distance between any two non cluster head nodes (non-CH's) of same cluster is negligible.

6. Energies stored in nodes are e1, e2, e3.... en.

7. After deployment nodes battery re-charge is not possible.

8. Communication channel is symmetrical between two nodes.

9. Nodes have cognitive radios in OFF state and have zero energy loss due to cognitive radios in CRSN.

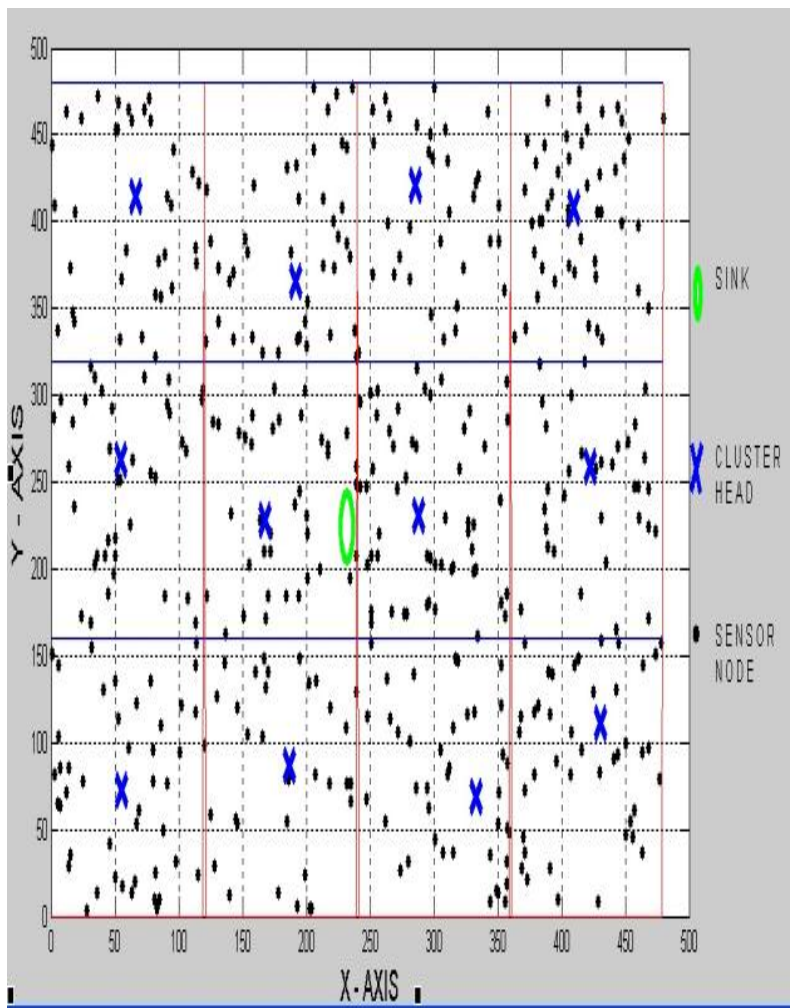

Fig 2: System Model for proposed Clustering Algorithm

As shown above in Fig.2 there is a xy plane. This xy plane is CRSN environment. This xy plane is divided into square boxes of almost equal size. These square boxes are formed by grouping sensor nodes. This group of nodes forms a cluster. The xy plane has black dots in it. These black dots are exactly sensor nodes which are positioned on xy plane. Initial energy associated to each sensor is different. Each cluster has a associated cross present inside it. This cross is actually a cluster head and all cluster heads have some min. energy threshold below which node can't act as a cluster head. Finally there is a rhombus present inside the xy plane. This is known as base station. Base station actually has record of each sensor node i.e. their location and energy etc.

\subsection{Radio Signal Propagation Model}

The energy consumption analysis is made using first order radio frequency energy consumption model. The energy consumption of transmitter is used for both radio communication and energy amplification. In case of receiver energy consumption is only used for radio communication. There are two models [6] used for energy consumption analysis depending on the threshold value of distance i.e.d0. If the distance between sender and receiver is less than $\mathrm{d} 0$ then free space model is used and if the distance is greater than $\mathrm{d} 0$ then multi path fading model is used. Thus energy consumed in sending $\mathrm{k}$ bits packet between two nodes having distance $\mathrm{d}$ between them is shown in equation (1) and energy consumed in receiving $\mathrm{k}$ bits packet between two nodes having distance $\mathrm{d}$ between them is shown in equation (2) .

$$
\begin{aligned}
& E_{T x}(k, d)=\left\{\begin{array}{ll}
E_{\text {elec }} * k+\varepsilon_{f s} * k * d^{2} & d \leq d_{0} \\
E_{\text {elec }} * k+\varepsilon_{\text {mp }} * k * d^{4} & d>d_{0}
\end{array}\right\} \\
& E_{R x}(k, d)=E_{\text {elec }} * k
\end{aligned}
$$

Where

$\mathrm{E}_{\text {elec }}=$ energy consumption per bit for transmitter and receiver circuit.

$\varepsilon_{\mathrm{fs}}=$ amplifier parameter for transmission in free-space model. $\varepsilon_{\mathrm{mp}}=$ amplifier parameter for transmission in multi-path model.

$\mathrm{d} 0=\sqrt{ }\left(\varepsilon_{\mathrm{fs} / \varepsilon \mathrm{mp})}\right.$.

If $\mathrm{d} \leq \mathrm{d} 0$, the channel approximates free-space model and the transmitter energy dissipation is in direct ratio to $\mathrm{d}^{2}$. On the other hand if $\mathrm{d}>\mathrm{d} 0$, the channel approximates multi-path model and transmitter energy dissipation is in direct ratio to $\mathrm{d}^{4}$.

\section{BECHR Clustering Algorithm}

Proposed BECHR clustering algorithm improves the overall lifetime of CRSN network by balancing the energy. It distributes the energy evenly between the clusters and also takes care of the problem that sensors within the cluster should be efficiently utilized so that they can't die in small time. BECHR clustering algorithm divides into two phases. First phase emphasizes on balancing the overall energy of network and the second phase emphasizing on how to improve the lifetime of each cluster.

Fig. 3 shows BECHR clustering. In this figure different colors of dots represent different clusters. 


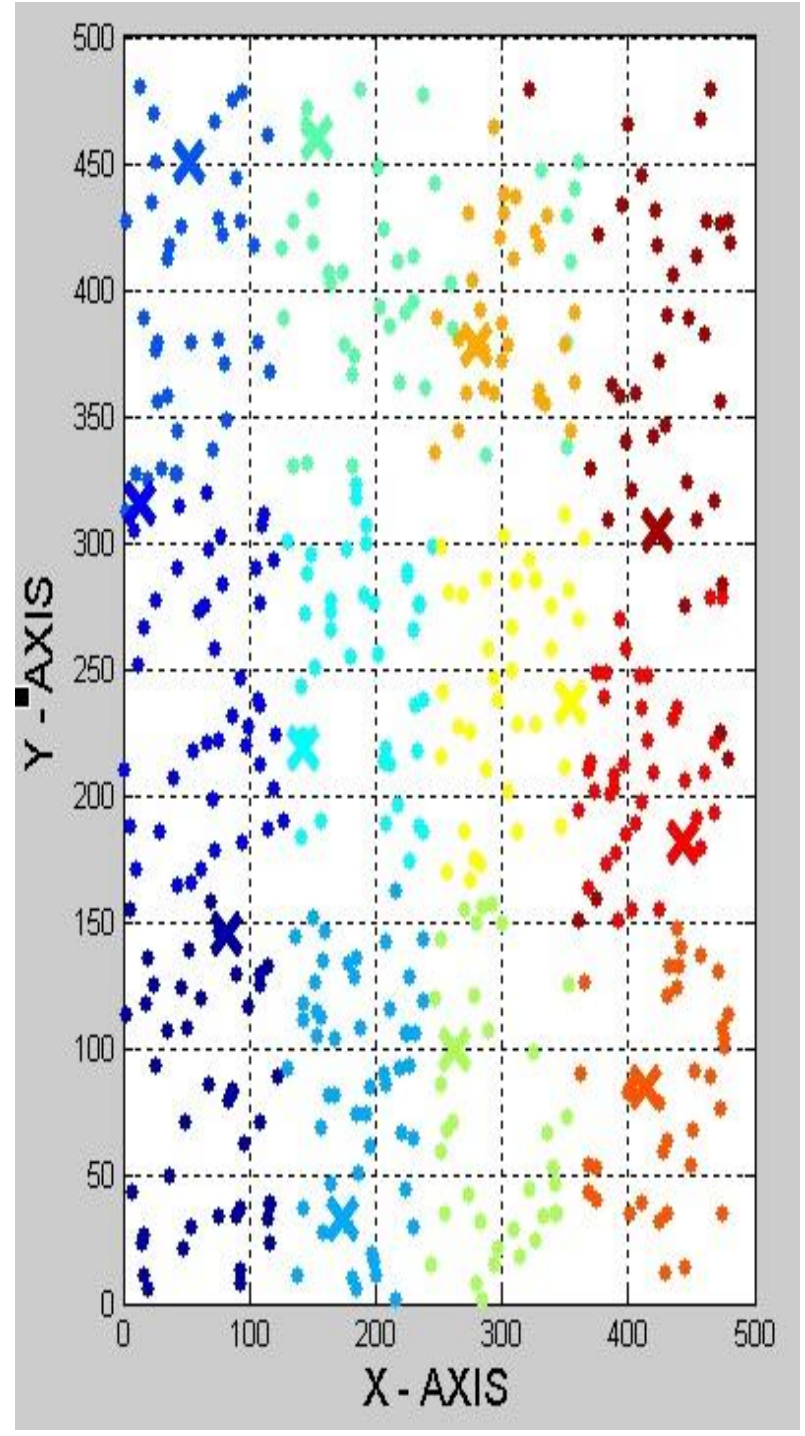

Fig 3: BECHR Clustering

\subsection{Initial Clustering phase}

In initial clustering phase energy efficient k-mean clustering is used which will improve the overall balance of energy between clusters. In original k-mean algorithm minimum distance is the criterion to group the nodes into a cluster formation but in proposed clustering algorithm min. Euclidian distance criterion along with energy balancing criterion is used. Following are the steps of initial clustering phase:

1. To start with, base station finds the initial points those are distributed uniformly in CRSN network and select them as initial cluster heads and group the nodes into a cluster according to minimum Euclidean distance criterion.

2. Now check if there is an energy imbalance between clusters then remove the border nodes of the cluster that has high energy than the threshold and attach it to neighbor cluster having low cluster energy than the threshold value. By doing so energy of each cluster becomes almost the same.

3. Select the node in each cluster which has highest energy level and assign this node a cluster head status.

The flow chart of initial clustering phase is shown in Fig.4.

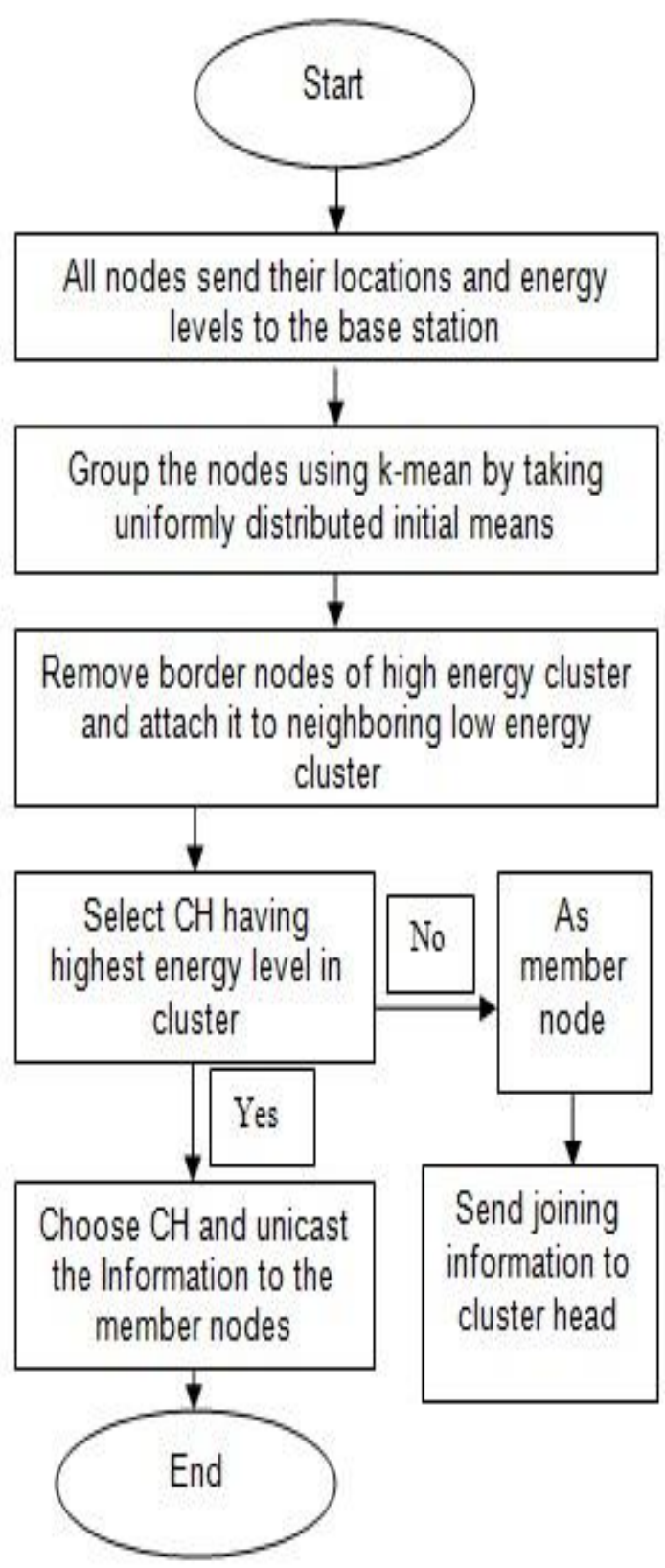

Fig 4: Initial Clustering Phase

\subsection{Steady Clustering Phase}

In steady clustering phase energy efficiency is improved by rotating cluster head of each cluster whenever its energy falls below threshold value. Instead of doing clustering of whole network again only new cluster head assignment is made and in doing so very less amount of energy is consumed. Following are the steps of steady clustering phase:

1. Nodes send their energy levels to the base station and BS compares it to threshold value.

2. If $\mathrm{CH}$ energy is lower than the threshold value then base station find sensor with the highest energy in that cluster and assign it a position of cluster head.

3. New cluster head broadcast $\mathrm{CH}$ status to the non $\mathrm{CH}$ nodes. Non $\mathrm{CH}$ nodes hear the advertisement of $\mathrm{CH}$ and attach themselves to new cluster head.

4. Create TDMA frame and send the data to cluster head and cluster head aggregate the data. 
5. Cluster head then sends data to base station.

The flow chart of steady clustering phase is shown in Fig.5.

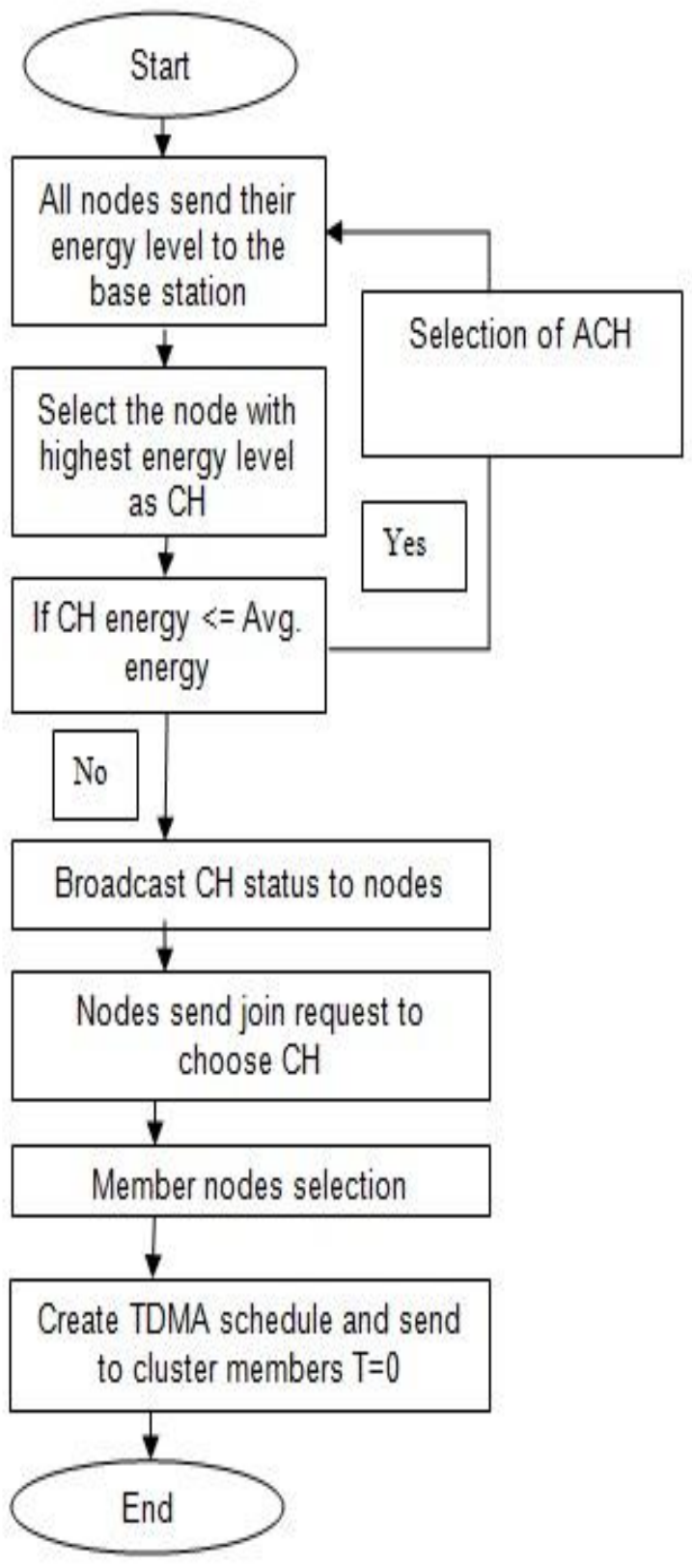

Fig 5: Steady Clustering Phase

\section{ADVANTAGES OF BECHR CLUSTERING}

If we compare BECHR clustering with clustering in LEACH protocol [7], the BECHR clustering has edge over existing clustering in LEACH protocol. Details of improvements are as follow:

1. Cluster head distribution in LEACH protocol is random throughout the network. While in BECHR clustering, cluster head are distributed uniformly.

2. Unlike LEACH protocol in which Cluster heads are selected at each round therefore energy exhausts not too quickly, proposed clustering in this paper is not round based clustering. In BECHR clustering scheme initially node with the highest energy is selected as a cluster head. When energy of that node falls below some threshold after some time only then the other node will replace old $\mathrm{CH}$ following the same principle. This will prevent the whole CRSN network to die too early.

3. Unlike LEACH protocol in which adaptive clustering that uses randomization to distribute energy throughout a network, BECHR clustering algorithm uses clustering technique in which every cluster has almost same energy. In this way the sensing results improves overall and faulty decisions are minimized as number of dead nodes are too small.

\section{SIMULATION AND RESULTS}

In this section performance of proposed BECHR clustering algorithm is compared with the existing clustering in LEACH protocol [7] and results are calculated using MATLAB and are shown in Fig.6 and Fig.7 respectively. In BECHR clustering scheme single base-station attempts to gather information from a number of cluster heads in the network area. The location of base station is randomly chosen. In this simulation system model has 480 nodes, those are distributed in 480 by 480 square meter area. There are two types of nodes present in simulation namely cluster head and non-cluster head nodes. All the nodes are stationary. For simulation initial energy for every node is 5 joules, both transfer energy loss and receive energy loss of $50^{*} 10^{\wedge}-8$ joules. Whenever a sensor transmits or receives a data packet, energy is being consumed from the residual energy of the sensor.

Simulation parameters are shown in the table below.

Table 1 Simulation Parameters

\begin{tabular}{|c|c|c|}
\hline $\begin{array}{c}\text { Serial } \\
\text { No. }\end{array}$ & Parameter & Value \\
\hline 1. & Initial node energy & 5 joules \\
\hline 2. & Nodes distribution & $\begin{array}{c}\text { Area } \\
480 \times 480\end{array}$ \\
\hline 3. & Base station position & $\begin{array}{c}\text { Coordinates } \\
(240,240)\end{array}$ \\
\hline 4. & Rounds for simulation & 500 \\
\hline 5. & Energy (free space) & $10 \times 10^{-12}$ joules \\
\hline 6. & Energy (multi path) & $0.0013 \times 10^{-12}$ joules \\
\hline 7. & Energy (transfer) & $50 \times 10^{-9}$ joules \\
\hline 8. & Energy (received) & $50 \times 10^{-9}$ joules \\
\hline 9. & Energy (aggregation) & $5 \times 10^{-9}$ joules \\
\hline
\end{tabular}

As can be seen in Fig.6, Number of dead nodes vs round graph, after 500 rounds out of 480 nodes almost 70 nodes died and that due to unbalanced cluster head selection and abnormal energy distribution between clusters. It can also be seen that dying of nodes had started from round 125 which shows clearly that the lifetime of network is be too small and therefore sensing results are not reliable with many faulty decisions.

On the other hand in Fig.6, Sum of energy vs round graph, it can be seen that the total amount of initial energy in the network is 2400 joules but after first 100 rounds total residual energy in network reaches below 2200 joules. In fact after 500 
rounds the total amount of residual energy reaches almost 1300 joules. Those clearly show that the Leach protocol is using network energy inefficiently.

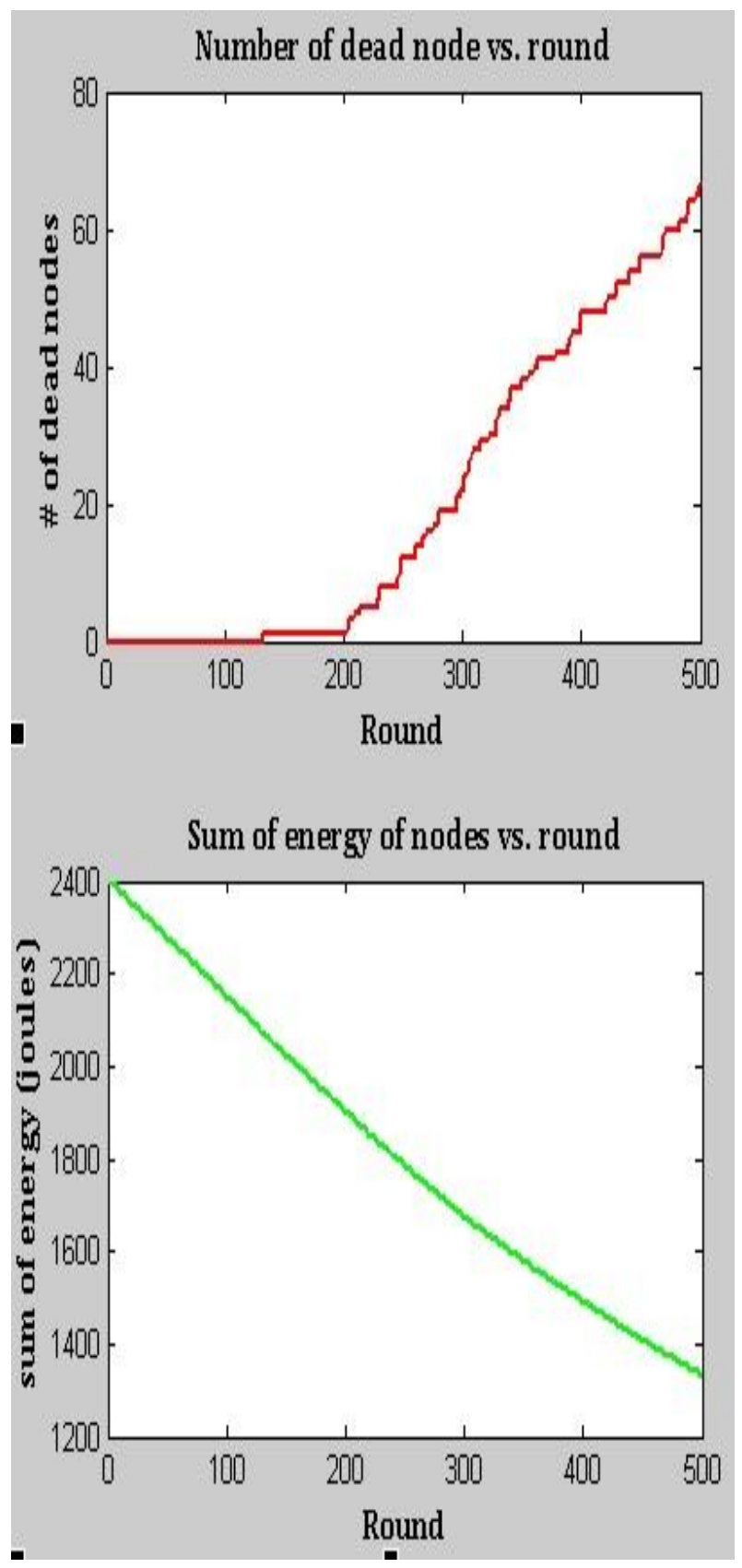

Fig 6: Dead Nodes \& Residual Energy for each round using LEACH Protocol

In Fig.7, Number of dead nodes vs round graph, it can be seen that after 500 rounds out of 480 nodes only 10 nodes died which clearly indicates balanced cluster head selection and uniform energy distribution between clusters. It can also be seen that dying of nodes had started from round 315 which shows clearly that the lifetime of network is longer than that of LEACH protocol and thus the sensing results improves comparatively with less faulty decisions.

On the other hand in Fig.7, Sum of energy vs round graph, it can be seen that the total amount of initial energy in the network is 2400 joules and after first 100 rounds total residual energy in network reaches almost 2350 joules. In fact after
500 rounds the total amount of residual energy reaches almost 2125 joules. Those clearly show that the BECHR clustering algorithm is using network energy efficiently.

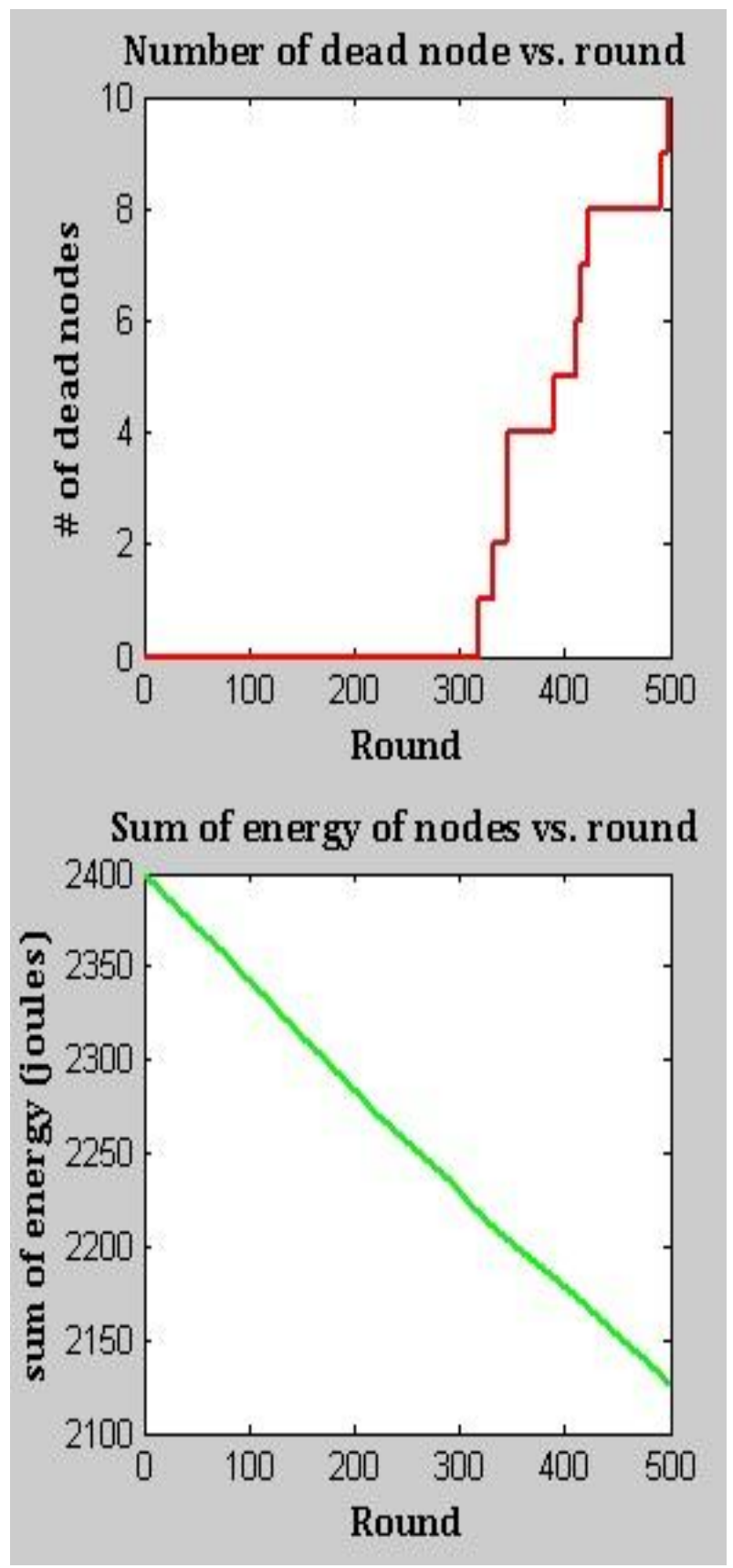

Fig 7: Dead Nodes \& Residual Energy for each round using BECHR Clustering Algorithm

The other difference due to which the lifetime of network improves is the balanced energy distribution among all clusters. The distribution of total energy between clusters for proposed BECHR clustering algorithm is shown in Fig.8. It can be seen that there is an approximately uniform distribution of energy between clusters. 


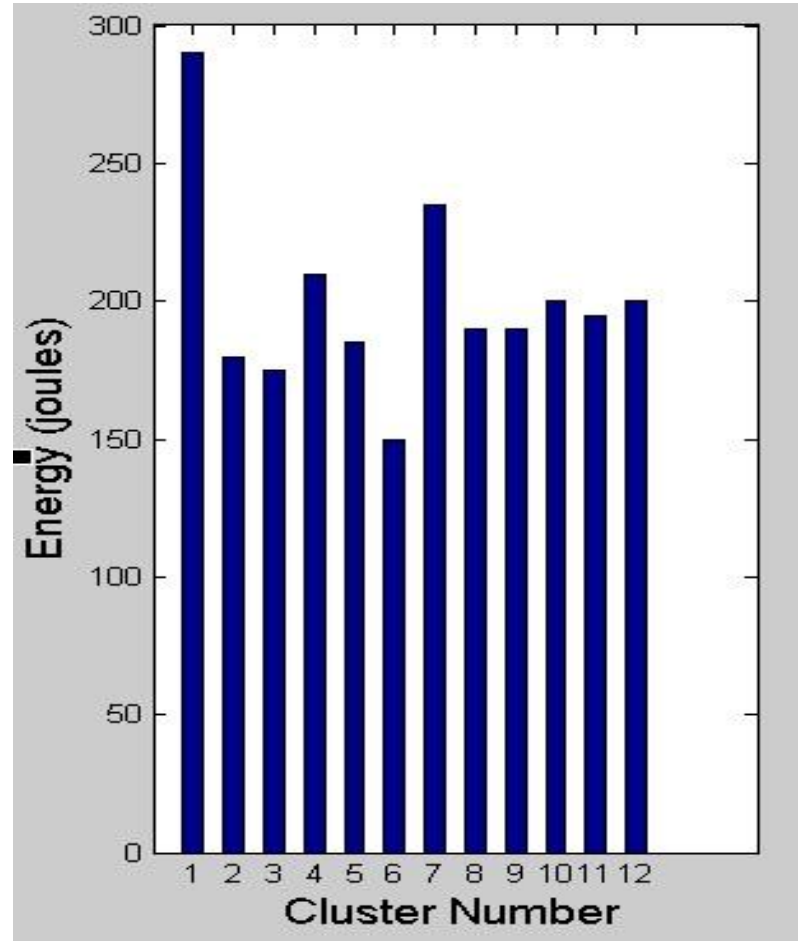

Fig 8: Energy distribution between Clusters for BECHR Clustering Algorithm

On the other hand, the distribution of total energy between clusters for existing clustering in LEACH protocol can be seen in Fig.9. It can be seen that there is a random distribution of energy between clusters.

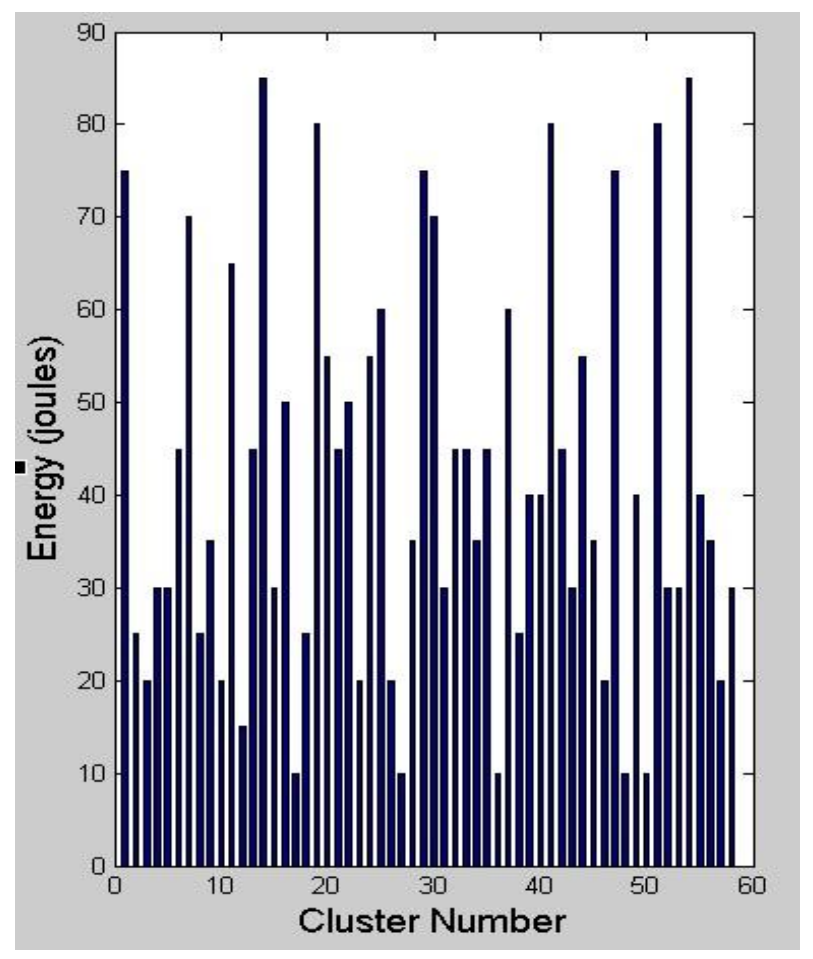

Fig 9: Energy distribution between Clusters for clustering in LEACH Protocol

\section{CONCLUSION}

A new BECHR clustering algorithm has been successfully presented and tested for life time of a network by checking the number of dead nodes and residual energy. In this paper it is presented that the proposed clustering algorithm is self organizing keeping energy balancing between clusters and also keep check on cluster head residual energy. Results show that in the proposed algorithm energy efficiency is improved and thus lifetime of network improved overall, reliable sensing results are achieved with less faulty decisions as numbers of dead nodes are much lower than the number of dead nodes of the LEACH protocol.

\section{ACKNOWLEDGMENTS}

The Authors wish to acknowledge the feedback from Dr. Najam Hassan and Dr. Mustafa Shakir and also like to thank Institute of Communication Technology (ICT), Faculty of Telecommunication Engineering for their technical support.

\section{REFERENCES}

[1] Ozgur B. Akan, Osman B. Karli, Ozgur Ergul, Cognitive Radio Sensor Networks. IEEE Network: The Magazine of Global Internetworking - Special issue title on networking over multi-hop cognitive networks, 2009, volume 23 , pp. 34-40.

[2] Abolarinwa J.A, SalawuN and Achonu A, Cognitive Radio-based Wireless Sensor Networks As Next Generation Sensor Network: Concept, Problems and Prospects, Journal of Emerging Trends in Computing and Information Sciences, ISSN 2079-8407, Vol.4, No.8 August 2013.

[3] Tata Jagannadha Swamy, Thaskani Sandhya and Dr. Garimella Ramamurthy, Energy Efficient Architecture to Cognitive Radio Wireless Sensor Networks, International Journal of Computer Networks and Wireless Communications (IJCNWC), ISSN: 2250-3501, Vol.2, No.6, December 2012.

[4] Ameer Ahmed Abbasi and Mohamed Younis, A survey on clustering algorithms for wireless sensor networks, Computer Communications, 2007, volume 30, pp. 28262841.

[5] Ossama Younis and Sonia Fahmy, Distributed Clustering for Scalable, Long-Lived Sensor Networks, Extended abstract in the $9^{\text {th }}$ Annual International Conference on Mobile Computing and Networking, 2003, ACM MobiCom, San Diego, CA.

[6] Renke Sun, Enjie Ding and Duan Zhao, The study of a wireless multimedia sensor network Self-Organization protocol for coal mine, 2nd International Conference on Computer Engineering and Technology, 2010, volume 4, Chengdu.

[7] Wendi Rabiner Heinzelman, Anantha Chandrakasan, and Hari Balakrishnan, Energy-Efficient Communication Protocol for Wireless Microsensor Networks, in Proceedings of the 33rd Hawaii International Conference on System Sciences, 2000, volume 8, pp. 3005-3014. 